\title{
Dexamethasone downregulates caveolin-1 causing muscle atrophy via inhibited insulin signaling
}

\author{
Young Hoon Son', Seok-Jin Lee', Ki-Baek Lee', Jin-Haeng Lee', Eui Man Jeong ${ }^{1,2}$, \\ Sun Gun Chung ${ }^{3}$, Sang-Chul Park ${ }^{1}$ and In-Gyu Kim ${ }^{1,2}$ \\ ${ }^{1}$ Department of Biochemistry and Molecular Biology, ${ }^{2}$ Institute of Human-Environment Interface Biology \\ and ${ }^{3}$ Department of Rehabilitation Medicine, Seoul National University College of Medicine, \\ 103 Daehak-ro, Jongno-Gu, Seoul 110-799, Korea
}

\author{
Correspondence \\ should be addressed \\ to I-G Kim \\ Email \\ igkim@plaza.snu.ac.kr
}

\begin{abstract}
Glucocorticoids play a major role in the development of muscle atrophy in various medical conditions, such as cancer, burn injury, and sepsis, by inhibiting insulin signaling. In this study, we report a new pathway in which glucocorticoids reduce the levels of upstream insulin signaling components by downregulating the transcription of the gene encoding caveolin-1 (CAV1), a scaffolding protein present in the caveolar membrane. Treatment with the glucocorticoid dexamethasone (DEX) decreased CAV1 protein and Cav1 mRNA expression, with a concomitant reduction in insulin receptor alpha $(\mathrm{IR} \alpha)$ and IR substrate 1 (IRS1) levels in $\mathrm{C} 2 \mathrm{C} 12$ myotubes. On the basis of the results of promoter analysis using deletion mutants and site-directed mutagenesis a negative glucocorticoid-response element in the regulatory region of the Cav1 gene was identified, confirming that Cav1 is a glucocorticoid-target gene. Cav1 knockdown using siRNA decreased the protein levels of IR and IRS1, and overexpression of Cav1 prevented the DEX-induced decrease in IR $\alpha$ and IRS1 proteins, demonstrating a causal role of Cav1 in the inhibition of insulin signaling. Moreover, injection of adenovirus expressing Cav1 into the gastrocnemius muscle of mice prevented DEX-induced atrophy. These results indicate that CAV1 is a critical regulator of muscle homeostasis, linking glucocorticoid signaling to the insulin signaling pathway, thereby providing a novel target for the prevention of glucocorticoid-induced muscle atrophy.
\end{abstract}
Key Words
- caveolin-1
- glucocorticoid
- insulin signaling
- muscle atrophy
- C2C12 myotube

Journal of Endocrinology (2015) 225, 27-37

\section{Introduction}

Glucocorticoids have diverse and tissue-specific effects on cellular metabolism. During metabolic stress, such as starvation, glucocorticoids promote degradation and suppress synthesis of muscle proteins in the skeletal muscle, thereby providing amino acids for glucose production in the liver (Kuo et al. 2013). Many pathological conditions, such as cancer, severe injury, sepsis, and long-term steroid therapy, cause persistent elevation of glucocorticoid levels, resulting in the development of muscle atrophy, in which a loss of muscle mass leads to muscle weakness. Thus, muscle atrophy is considered to be a prognostic factor for underlying diseases (Schakman et al. 2013).

Glucocorticoids cause muscle atrophy by upregulating the expression of two muscle-specific ubiquitin ligases, MuRF1 and MAFbx/Atrogin1 (Glass 2010). MuRF1 ubiquitinates troponin I, myosin heavy chain, and myosin binding protein $\mathrm{C}$, and thus induces their proteasomal degradation (Clarke et al. 2007, Polge et al. 2011). In contrast,

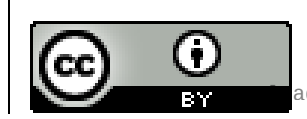


MAFbx/Atrogin1 suppresses protein synthesis through the ubiquitination and degradation of eIF3 $\mathrm{f}$ and MyoD, key factors regulating translation and transcription in muscle respectively (Attaix \& Baracos 2010). Results described in recent reports have indicated that glucocorticoids regulate MuRF1 and MAFbx/Atrogin1 through KLF15, a transcription factor involved in muscle catabolism via cooperation with FoxO1 (Attaix \& Baracos 2010).

Glucocorticoids also suppress muscle protein synthesis through inhibition of insulin/insulin-like growth factor 1 (IGF1) signaling. Glucocorticoid-induced REDD1 inhibits mTOR by stabilizing the TSC1-TSC2 complex, resulting in decreased phosphorylation of both eIF4Ebinding protein 1 and ribosomal protein S6 kinase 1, two critical factors regulating translation in the ribosome (Attaix \& Baracos 2010). In addition, glucocorticoids inhibit PI3K/AKT signaling through the reduction in the levels of insulin receptor substrate 1 (IRS1) protein, possibly by accelerating IRS1 degradation (Koh et al. 2013). Although the molecular mechanism regulating the glucocorticoid-mediated reduction in the insulin receptor alpha (IR $\alpha)$ and IRS1 levels is not fully understood, crosstalk between glucocorticoid and insulin/IGF1 signaling could play a crucial role in the maintenance of muscle mass.

Caveolin (CAV) is the major protein component of caveolae, which are invaginations of the plasma membrane found in most cell types. CAV functions as a scaffolding protein within the caveolae, where it regulates signaling pathways through its interaction with diverse membrane proteins, such as G-proteins or receptors (Shimizu et al. 2011). There are three CAV isoforms expressed in muscle (Kim et al. 2009). CAV3 is expressed predominantly in muscle, where it forms a homo-oligomer in the T tubule system and plays a role in muscle development and sarcolemma repair under mechanical stress. Mutations in the $C A V 3$ gene cause several degenerative muscle diseases in humans, including limb-girdle muscular dystrophy, rippling muscle disease, and distal myopathy (Koh et al. 2013). CAV1 is expressed in the muscle and lung, and forms a homo-oligomer or a hetero-oligomer with CAV2 (Hansen \& Nichols 2010). Cav1-deficient mice have impaired nitric oxide and calcium signaling, resulting in dilated cardiomyopathy and thickening of the alveolar septa in the lung. In humans, CAV1 mutations cause generalized lipodystrophy, insulin resistance, and hypertriglyceridemia (Gazzerro et al. 2010). Moreover, we have demonstrated that the level of CAV1 in skeletal muscle is related to insulin sensitivity in vitro and in vivo (Oh et al. 2008), indicating that CAV1 may regulate insulin signaling. Notably, Cav1-deficient mice have a lean phenotype
(Razani et al. 2002). Furthermore, CAV1 expression is dependent on androgens and exercise (Baehr et al. 2011, Oh et al. 2011). These data from Cav-deficient mice indicate that Cav1 may play a key role in glucocorticoid regulation of the insulin pathway, thereby contributing to muscle atrophy.

In this study, we evaluated the role of CAV isoforms in a glucocorticoid-induced muscle atrophy model, and found that dexamethasone (DEX) treatment decreased Cav1, but not Cav2 and Cav3, expression. Downregulation of Cav1 suppressed expression of IR $\alpha$ and IRS1, thereby increasing MAFbx/Atrogin1 and MuRF1 expression. Conversely, overexpression of Cav1 prevented DEX-induced muscle atrophy in vitro and in vivo. Our results indicate that Cav1 mediates glucocorticoid-induced suppression of insulin signaling.

\section{Methods and materials}

\section{Cell culture}

C2C12 cells (American Type Culture Collection, Manassas, VA, USA) were cultured in DMEM, supplemented with $10 \%$ fetal bovine serum (FBS), $100 \mathrm{U} / \mathrm{ml}$ penicillin, and $100 \mu \mathrm{g} / \mathrm{ml}$ streptomycin. To induce myoblast fusion and differentiation, cells were grown to about $80 \%$ confluency, and the medium was replaced with DMEM containing $2 \%$ FBS. The medium was changed every 2 days. After 4 days, the $\mathrm{C} 2 \mathrm{C} 12$ myotubes were treated with various doses of DEX $(100 \mathrm{nM}, 1 \mu \mathrm{M}, 10 \mu \mathrm{M}$, and $100 \mu \mathrm{M})$ for the indicated times to induce myotube atrophy. To confirm the nuclear translocation of p-GR after DEX treatment, cells were incubated with anti-pGR antibody (for phosphorylation at $\mathrm{S}^{211}$ ) and FITC-conjugated secondary antibody. The fluorescently stained cells were photographed using a LSM501META confocal microscope (Zeiss, Oberkochen, Germany). The cells were used at low passage (under ten passages) for all experiments to maintain their differentiation potential (Menconi et al. 2008).

\section{Measurement of myotube diameter}

After differentiation, C2C12 myotubes were stained using a modified PAS staining kit (Merck). C2C12 myotubes were treated for $5 \mathrm{~min}$ with periodic acid, followed by washing with PBS for $3 \mathrm{~min}$. After washing with PBS, Schiff's reagent was added to the cells for $15 \mathrm{~min}$ at room temperature. After another wash with PBS, nuclei were stained for 2 min with Gill III solution. C2C12 myotubes were mounted in Neo-Mount solution and photographed under a phase-contrast microscope at $100 \times$ magnification.

Published by Bioscientifica Ltd 
The diameters of 50 myotubes were measured in at least ten random fields using ImageJ Software (National Institutes of Health, Frederick, MD, USA). The results are expressed as percentages of the diameter of the control myotubes.

\section{Promoter analysis}

The Cav1 promoter region (GenBank accession number NC_000072.6) spanning positions -2000 to +300 was amplified from genomic DNA using PCR (Roche Molecular Biochemicals). Four deletion mutants of the Cav1 promoter, spanning from positions $-2000,-1037$, -500 , and -100 to +300 , were generated using PCR and cloned into pGL2-Basic (Promega) for the luciferase assays. Site-directed mutagenesis was performed following the manufacturer's instructions (Stratagene, La Jolla, CA, USA). All constructs were verified by DNA sequencing (Applied Biosystems 3730xl DNA Analyzer, Life Technologies). To define the transcriptional upstream region of the Cav1 gene, we used FASTA data from the NCBI (http://www.ncbi. nlm.nih.gov/nuccore/NC_000072.5). Putative glucocorticoid response element (GRE) sequences were identified in this region (positions -2000 to +300 ) of the Cav1 gene using http://www.cbil.upenn.edu/cgibin, http://alggen.lsi. upc.es/cgibin/promo_v3, and http://www.gene-regulation. com/cgi-bin.

\section{Transfection and luciferase assays}

C2C12 cells $\left(2 \times 10^{4}\right)$ were seeded into a 12-well plate and cultured for $24 \mathrm{~h}$. The cells were then transfected with reporter constructs using Lipofectamine 2000 (Invitrogen, Life Technologies) according to the manufacturer's instructions. After reaching confluence, the cells were cultured in differentiation medium for 4 days and then treated with $1 \mu \mathrm{M}$ DEX for $24 \mathrm{~h}$. After preparation of the myotube lysates, luciferase activity was assayed using a dual-luciferase reporter assay system (Promega) and normalized to Renilla luciferase activity. The results are expressed as the mean \pm s.D. of at least five independent experiments.

\section{Western blot analysis}

We prepared lysates with RIPA buffer and performed SDSPAGE as described previously (Menconi et al. 2008). An equal amount of protein was transferred to nitrocellulose membrane and probed with the following antibodies: MAFbx/Atrogin1 (ECM Bioscience, Versailles, KY, USA), MuRF1 (GeneTax, Irvine, CA, USA), CAV1, CAV2, CAV3
(BD, Franklin Lakes, NJ, USA), glucocorticoid receptor (GR), lamin, MyoD (Santa Cruz Biotechnology), COX1, EIF3F (Abcam, Cambridge, MA, USA), p-GR (phosphorylated at $S^{211}$ ) (Cell Signaling Technology, Danvers, MA, USA), $\alpha$-tubulin (Sigma-Aldrich), IR $\alpha$ (Santa Cruz Biotechnology), and IRS1 (Cell Signaling Technology). Secondary antibodies were added for $1 \mathrm{~h}$ at room temperature. The antibody-antigen complexes were detected using an ECL Kit (Pierce, Rockford, IL, USA). The signal intensity was determined using an LAS-3000 image reader (Fujifilm, Tokyo, Japan). To inhibit proteolysis, C2C12 myotubes were pretreated with MG132 $(20 \mu \mathrm{M})$ for $1 \mathrm{~h}$ before DEX treatment.

\section{Real-time quantitative RT-PCR}

Total RNA was isolated from C2C12 myotubes using the TRI reagent (Invitrogen; Menconi et al. 2008), and the expression levels of MAFbx/Atrogin1, MuRF1, Cav1, and Gapdh mRNA were assessed by real-time RT-PCR using an iCycler RT-PCR instrument (Bio-Rad). For each sample, 25 ng total RNA were subjected to RT-PCR (in duplicate) according to the protocol provided by the manufacturer. The following specific primers were used:MAFbx/Atrogin1, 5'-CTCTGTACCATGCCGTTCCT-3', (forward) and 5'-GGCTGCTGAACAGATTCTCC-3' (reverse); MuRF1, 5'-TGTCTGGAGGTCGTTTCCG-3' (forward) and 5'-TGCCGGTCCATGATCACTT-3' (reverse); Cav1, 5'-ACG ATGTCTGGGGGCAAATAC-3' (forward) and 5'-TCATATCTCTTTCTGCGTGC-3' (reverse); and Gapdh, $5^{\prime}$-CCACCCATGGCAAATTCCATGGCA-3' (forward) and 5'-TCTAGACGGCAGGTCAGGTCCACC-3' (reverse).

\section{siRNA knockdown of Cav1}

siRNA specific for Cav1 was purchased from Santa Cruz Biotechnology. siRNA (10 nM) was transfected into C2C12 myotubes for $10 \mathrm{~min}$ using the G-fectin transfection protocol (Genolution Pharmaceuticals, Seoul, Korea).

\section{Animals}

Male C57BL/6 mice (8 months old) were purchased from the Osong Aging Animal Laboratory (Cheongju, Chungcheong bukdo, Korea). Mice were given ad libitum access to food and water and were maintained on a $12 \mathrm{~h}$ light: $12 \mathrm{~h}$ darkness cycle. All animal procedures were approved by the Institutional Animal Care and Use Committee of the Seoul National University College of Medicine. The animals were randomly assigned to four groups $(n=5$ for each group). The control group received saline, the DEX

Published by Bioscientifica Ltd 
group received $5 \mathrm{mg} / \mathrm{kg}$ of DEX every other day by i.p. injection for 12 days. The DEX-AdGFP and DEX-AdCav1 groups received adenovirus expressing GFP or CAV1 $\left(3 \times 10^{11}\right.$ p.f.u.), respectively, in $10 \mu \mathrm{l}$ of saline by daily injection into the gastrocnemius muscle at eight sites for 3 days. The animals were anesthetized using isoflurane before adenovirus administration. After the animals were killed by cervical dislocation, the gastrocnemius muscle was obtained for western blot analysis and morphological evaluation.

\section{Preparation of adenovirus}

The adenoviral vectors and infective recombinant adenovirus expressing Cav1 used in this study have been described previously (Oh et al. 2008). In brief, infective recombinant adenovirus was made using the AdEasy system. Cav1 cDNA was inserted into the pAdTRACKCMV plasmid (Invitrogen) and transfected into Escherichia coli BJ5183 with the adenovirus containing the pAdEasy-1 plasmid by electroporation. Recombinant viruses were selected with kanamycin. The virus stocks were amplified in HEK293 cells on $15 \mathrm{~cm}$ plates and purified using BD Adeno-X Purification Kits (Clontech).

\section{Measurement of muscle fiber cross-sectional area}

Muscle sections $(10 \mu \mathrm{m})$ were obtained and mounted on glass slides. The sections were fixed for 2 min with acetone and stained with hematoxylin and eosin (H\&E). Images of muscle fiber cross-sectional area (CSA) were visualized at $200 \times$ magnification using a light microscope and captured with a microscope camera (Zeiss). At least 200 fibers per muscle were randomly chosen and analyzed using ImageJ.

\section{Statistical analyses}

All data are presented as mean \pm s.D. Differences between two variables were assessed using the unpaired Student's $t$-test or two-way ANOVA. A difference was considered significant if the $P$ value was $<0.05$. All statistical calculations were performed using GraphPad Prism version 5.0 (GraphPad Software, San Diego, CA, USA).

\section{Results}

\section{DEX negatively regulates Cav1 expression}

To examine the role of caveolins in glucocorticoidinduced muscle atrophy, we used DEX-treated C2C12 myotubes as an in vitro model (Menconi et al. 2007, Schakman et al. 2008). C2C12 myotubes were treated with DEX at several concentrations ( $100 \mathrm{nM}, 1 \mu \mathrm{M}, 10 \mu \mathrm{M}$, and $100 \mu \mathrm{M})$ for various periods. Results of western blot analyses indicated that DEX treatment induced maximal GR expression at a concentration of $100 \mathrm{nM}$ (Fig. 1A), and promoted the translocation of the GR into the nucleus (Fig. 1B). Protein levels of MAFbx/Atrogin1 and MuRF1, two markers of muscle atrophy, were increased in a doseand time-dependent manner, and peaked following treatment with DEX for $24 \mathrm{~h}$ at $1 \mu \mathrm{M}$ (1.5- and 5.2-fold respectively; Fig. 1A). DEX-induced changes in protein abundance were confirmed by real-time PCR. Transcript levels of MAFbx/Atrogin1 (2.6-fold, $P<0.05$ ) and MuRF1 (6.8-fold, $P<0.001)$ were increased by exposure to DEX (Fig. 1C). In contrast, protein levels of the muscle differentiation markers MyoD and EIF3F, as well as insulin signaling molecules IR $\alpha$ and IRS1, were decreased in a dose- and time-dependent manner by DEX treatment (Fig. 1A). Furthermore, DEX treatment $(1 \mu \mathrm{M}, 24 \mathrm{~h})$ reduced the mean diameter of myotubes by $20 \%$ in comparison with the control cells (Fig. 1D).

Under the same experimental conditions, we found that the level of CAV1 protein in C2C12 myotubes was decreased in a dose-dependent manner by DEX ( 0.55 -fold, $1 \mu \mathrm{M}$ for $24 \mathrm{~h}$ ), whereas the levels of CAV2 and CAV3 were not altered (Fig. 1A and Supplementary Fig. 1, see section on supplementary data given at the end of this article). DEX regulates the transcription of many genes in a positive or negative way. To test whether DEX regulates Cav1 expression at the transcriptional level, we performed realtime PCR and found that Cav1 mRNA abundance was significantly decreased in myotubes treated with DEX (Fig. 1C). Moreover, MG132 had no effect on CAV1 protein abundance after DEX treatment (results not shown), indicating that CAV1 was downregulated by DEX through transcriptional suppression, and not through Atrogin1- or MuRF1-mediated proteolytic degradation.

To further confirm the transcriptional regulation of Cav1 by DEX, we generated promoter deletion constructs based on a sequence analysis that revealed approximately 30 putative GREs within a 2-kbp region upstream of the transcriptional start site of Cav1. The deletion constructs were fused with a luciferase reporter and transfected into the $\mathrm{C} 2 \mathrm{C} 12$ myotubes. The reporter assay revealed that DEX caused a $50 \%$ reduction in luciferase activity in comparison with the control cells, but DEX failed to exhibit a suppressive effect after deletion of a region spanning from positions -500 to -100 of the Cav1 gene (Fig. 2A). To determine the specific GRE responsible for the

Published by Bioscientifica Ltd 
A

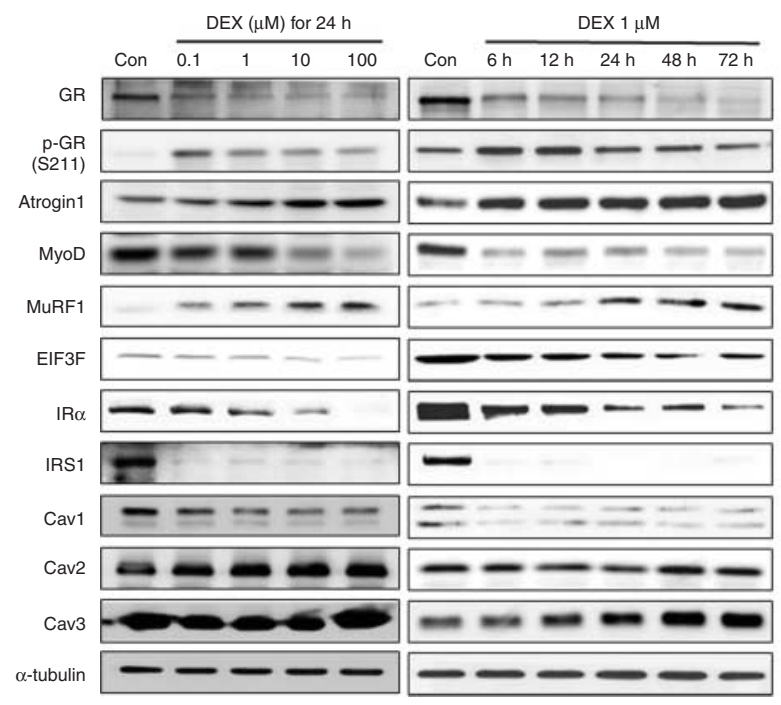

B
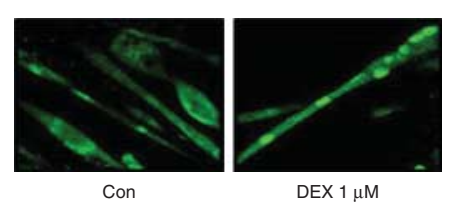

C
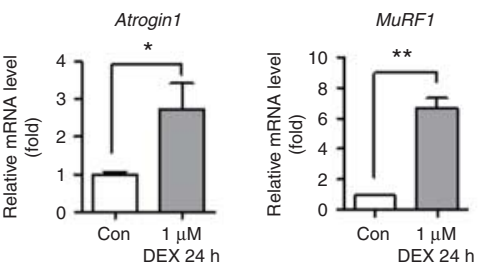

D
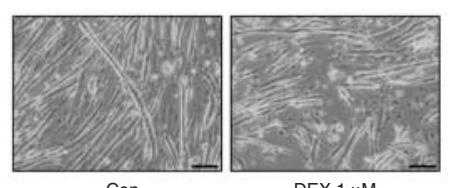

$\operatorname{DEX} 1 \mu \mathrm{M}$
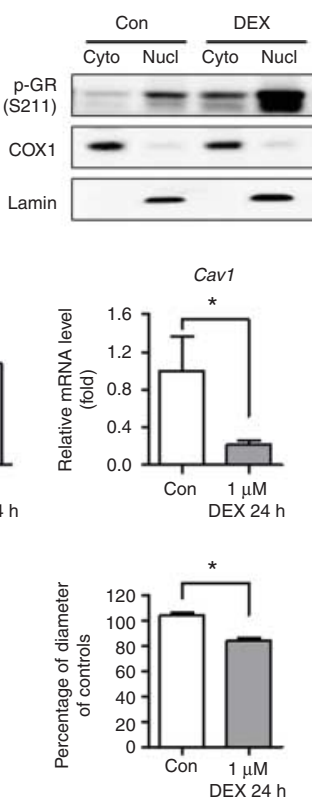

\section{Figure 1}

Dexamethasone suppresses caveolin-1 expression at the transcriptional level in C2C12 myotubes. (A) Effect of DEX treatment on the expression of caveolins. C2C12 myotubes were treated with DEX $(100 \mathrm{nM}, 1 \mu \mathrm{M}, 10 \mu \mathrm{M}$, and $100 \mu \mathrm{M})$ at various time points. Cell lysates were analyzed by western blotting using antibodies against glucocorticoid receptor (GR), glucocorticoid receptor phosphorylated at $S^{211}$ (p-GR), MAFbx/Atrogin1, MyoD, MuRF1, EIF3F, insulin receptor alpha (IR $\alpha$ ), IRS1, caveolin-1 (CAV1), caveolin-2 (CAV2), and caveolin-3 (CAV3). (B) Nuclear translocation of p-GR following DEX treatment ( $1 \mu \mathrm{M}, 24 \mathrm{~h})$ was monitored by confocal microscopy and western blot analysis. Cox 1 and lamin were used as markers

negative regulation of Cav1 gene, we generated six GRE mutants located in this region, and found that the sitespecific mutation (CAGAG to CACAG) of a GRE located at position -387 produced mutants that failed to respond to DEX (Fig. 2B and Supplementary Fig. 2, see section on supplementary data given at the end of this article). These results indicate that DEX suppresses Cav1 expression in C2C12 myotubes through a negative regulatory GRE.

\section{Cav1 mediates DEX-induced suppression of insulin signaling}

CAV1 is a scaffolding protein that recruits components of various signaling pathways to the caveolar membrane, thereby activating or repressing signaling (Boscher \& Nabi 2012). To test whether CAV1 is involved in the regulation of signaling pathways required for muscle growth or protein degradation, we first examined the effect of Cav1 knockdown on insulin signaling. C2C12 myotubes were treated with Cav1-specific siRNA for $24 \mathrm{~h}$, and the protein levels of IR $\alpha$ and IRS1 were evaluated. Cav1 siRNA

for the cytosolic (Cyto) and nuclear (Nucl) fractions respectively. (C) Total RNA from C2C12 myotubes treated with $1 \mu \mathrm{M}$ of DEX for $24 \mathrm{~h}$ was analyzed by quantitative RT-PCR for MAFbx/Atrogin1, MuRF1, and Cav1 $(n=3)$.

The data are given as mean \pm s.D. ${ }^{*} P<0.01$. (D) Myotubes treated with DEX $(1 \mu \mathrm{M}, 24 \mathrm{~h})$ were stained with PAS and photographed. The diameter of the myotubes from randomly selected fields was measured using ImageJ. The diameters are expressed as percentages of the diameter of the control myotubes $(n=50)$. Results are expressed as mean \pm s.D. Scale bars: $100 \mu \mathrm{m}$. A full colour version of this figure is available at http://dx.doi.org/10.1530/ JOE-14-0490.

treatment resulted in a $40 \%$ reduction in CAV1 protein expression in comparison with control-siRNA-treated myotubes. In cells treated with Cav1-specific siRNA, protein levels of IR $\alpha$ and IRS1 were significantly decreased by up to 90 and $80 \%$, respectively, in comparison with the control siRNA-treated myotubes (Fig. 3A and B).

Reduced insulin signaling decreases AKT activity, and thereby leads to a marked increase in nuclear FoxO. As increased nuclear FoxO promotes E3 ligase expression (Zhao et al. 2009), we next examined E3 ligase protein levels in Cav1 siRNA-treated myotubes. Results of western blot analyses indicated that protein levels of MAFbx/ Atrogin1 and MuRF1 were significantly increased by three- and tenfold respectively (Fig. 3A and B). Moreover, Cav1-knockdown caused a 20\% reduction in the diameter of myotubes in comparison with those treated with the control siRNA (Fig. 3C and D). These results indicate that CAV1 mediates DEX-induced suppression of insulin signaling, thereby increasing E3 ligase expression.

To confirm the causal relationship between CAV1 expression and insulin signaling, we tested whether CAV1

Published by Bioscientifica Ltd 


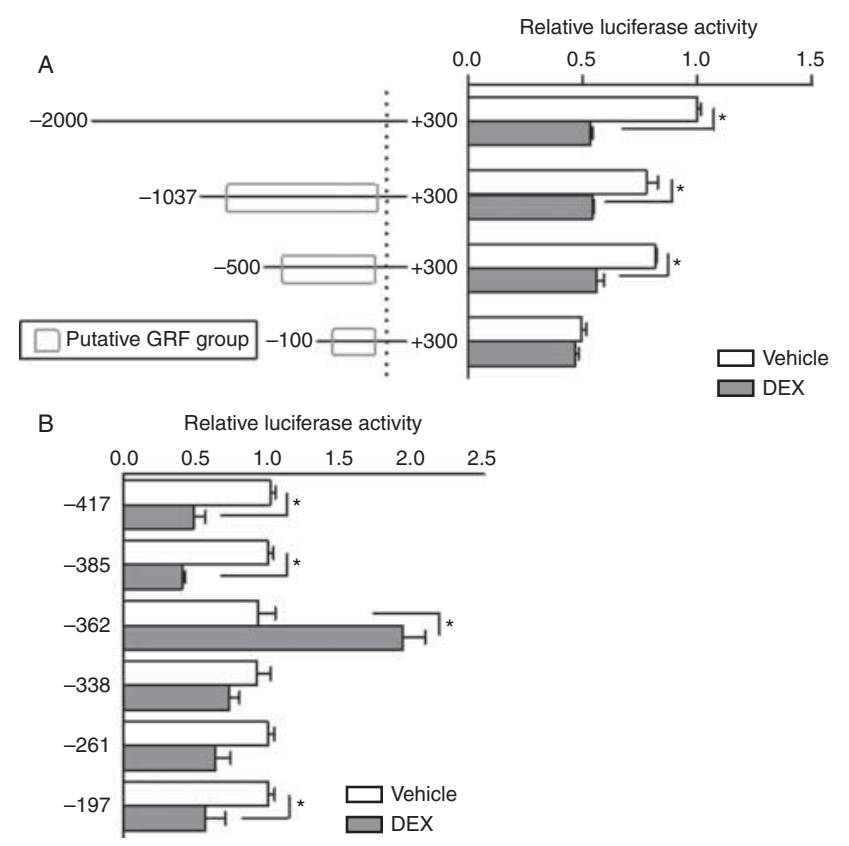

Figure 2

Identification of a negative glucocorticoid-responsive element in the mouse caveolin-1 promoter. (A) Luciferase reporter activity of deletion constructs of the mouse Cav1 promoter. $\mathrm{C} 2 \mathrm{C} 12$ myotubes transfected with deletion constructs were treated with $1 \mu \mathrm{M}$ of DEX for $24 \mathrm{~h}$. Cell lysates were assayed for luciferase activity. (B) The effect of site-specific mutagenesis of GREs (CAGAG to CACAG) on reporter activity. Luciferase activity is expressed relative to that for vehicle-treated myotubes $(n=5)$. Data are expressed as mean \pm s.D.

overexpression could suppress E3 ligase expression through upregulation of insulin signaling. To this end, adenoviruses expressing Cav1 (AdCav1) and Gfp (AdGfp) were generated and infected into the cultured $\mathrm{C} 2 \mathrm{C} 12$ myotubes. Western blot analysis showed that AdCav1 increased the protein level of CAV1 1.7-fold in comparison with AdGFP-infected myotubes. Under the same experimental conditions, protein levels of IR $\alpha$ and IRS1 were elevated three- and twofold respectively. Concomitantly, protein levels of MAFbx/Atrogin1 and MuRF1 were decreased by 30 and $70 \%$ respectively (Fig. $4 \mathrm{~A}$ and B). Moreover, when these cells were treated with DEX, Cav1-overexpression prevented more than $80 \%$ of the DEX-induced reduction in IR $\alpha$ and IRS1 expression, as well as $25-60 \%$ of the DEX-induced increase in MAFbx/ Atrogin1 and MuRF1 expression (Fig. 4A and B). In addition, when the diameters of the myotubes were compared, Cav1 overexpression prevented up to $20 \%$ of the DEX-induced reduction in mean diameter (Fig. $4 \mathrm{C}$ and D). These results obtained from the siRNA and adenoviral overexpression systems indicate that CAV1 is a key regulator of DEX-induced insulin signaling.

\section{Cav1 overexpression restores muscle loss in DEX-treated mice}

We next sought to extend these results from a cellular model to an in vivo model of DEX-induced muscle atrophy by assessing whether Cav1 overexpression could restore muscle loss induced by DEX treatment. C57BL/6 mice received intraperitoneal injections of DEX ( $5 \mathrm{mg} / \mathrm{kg}$ body weight) every other day for 12 days, and were then treated with a daily injection of AdCav1 or AdGFP virus to the gastrocnemius muscle in eight places for 3 days.

DEX treatment and adenovirus injection did not significantly affect the body weight of the mice during the treatment period (Fig. 5A). AdCav1 injection restored the weight of the gastrocnemius muscle and the ratio of gastrocnemius to body weight after DEX treatment, but these results were not statistically significant (Fig. 5B). Thus, to support these findings at the tissue level, we compared the size of myofibers in the gastrocnemius muscle by measuring the CSA after H\&E staining (Fig. 5C). In the gastrocnemius muscle, DEX treatment decreased the number of myofibers with a larger CSA in comparison with that of saline-treated mice. In contrast, $A d C a v 1$ injection into the gastrocnemius muscle of DEX-treated mice increased the number of myofibers with a larger CSA in comparison with that of mice that received injections of virus encoding AdGFP (Fig. 5D), indicating that Cav1 overexpression reversed the DEX-induced myofiber size reduction in vivo.

To confirm the role of CAV1 in DEX-induced muscle atrophy at the molecular level, we evaluated the levels of various proteins involved in insulin signaling in the gastrocnemius muscle. Results of western blot analysis indicated that DEX treatment decreased IR $\alpha$ and IRS1 protein levels, resulting in increased expression of E3 ubiquitin ligases (MAFbx/Atrogin1 and MuRF1) and decreased expression of MyoD and EIF3F in the gastrocnemius muscle. Cav1 overexpression induced by AdCav1 injection reversed the effects of DEX on the abundance of these insulin-related proteins. It is of note that Cav1 overexpression had no effect on expression of Cav2 or Cav3 in the gastrocnemius muscle (Fig. 5E and F). Collectively, these results confirmed that DEX induced muscle atrophy through downregulation of CAV1.

\section{Discussion}

Skeletal muscle mass is maintained through a balance of protein synthesis and degradation regulated by a number of signaling pathways, including the insulin/IGF, myostatin, and NF-kB pathways (Bonaldo \& Sandri 2013). The insulin/IGF-PI3K-AKT pathway is a muscle regulation

Published by Bioscientifica Ltd 


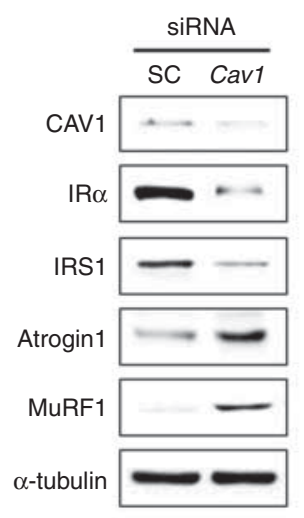

C

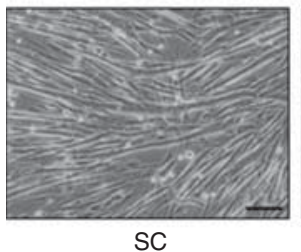

B

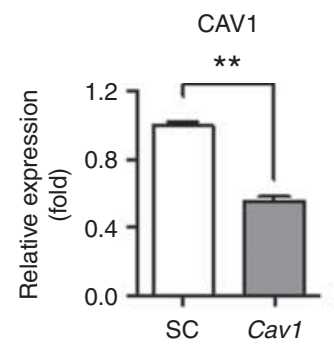

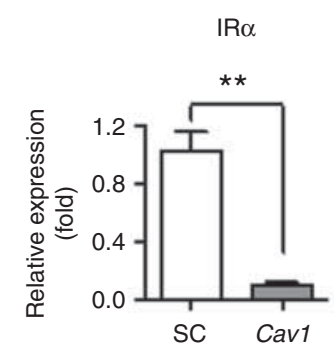

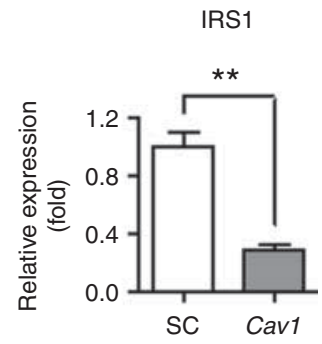

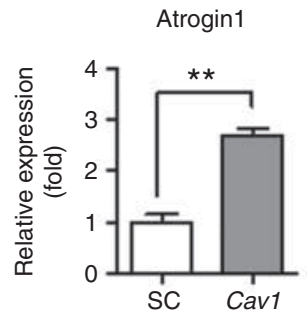

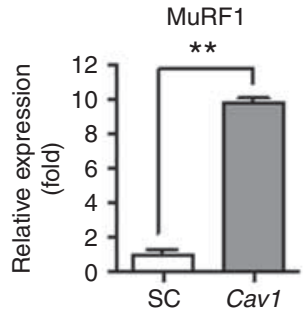

\section{Figure 3}

Caveolin-1 knockdown decreases C2C12 myotube diameter through downregulation of IR $\alpha$ and IRS1. (A) Western blot analyses of $\mathrm{C} 2 \mathrm{C} 12$ myotubes treated with Cav1 siRNA or scramble (SC) siRNA using antibodies against IR $\alpha$, IRS1, MAFbx/Atrogin1, and MuRF1. (B) Relative band intensities from three independent experiments were estimated by densitometric quantification. Data are presented as mean \pm s.D. ${ }^{*} P<0.01$.

pathways that promotes protein synthesis and suppresses protein degradation, resulting in an increase in the size of myofibrils (Glass 2010). The results presented here indicated that DEX treatment suppresses Cav1 expression in muscle cells, causing a decrease in IR $\alpha$ and IRS1 and an increase in MAFbx/Atrogin1 and MuRF1 protein expression. Conversely, overexpression of Cav1 in mouse muscle prevented DEX-induced muscle loss. These results indicate that glucocorticoids effectively inhibit the anabolic effects of insulin through negative regulation of upstream components of the signaling pathway. Moreover, the results from the Cav1 siRNA and overexpression assays indicate that regulation of insulin signaling by glucocorticoids is a crucial point of control in the maintenance of muscle homeostasis, especially under conditions of catabolic stress. Thus, downregulation of Cav1 gene expression is a new mechanism by which glucocorticoids inhibit the insulin signaling pathway.
(C) Representative photographs of $\mathrm{C} 2 \mathrm{C} 12$ myotubes treated with Cav1 siRNA or scramble siRNA. Scale bars: $100 \mu \mathrm{m}$. (D) Cells were stained with PAS and photographed. The diameter of myotubes from randomly selected fields was measured using Image J. Data are expressed as percentages of the diameter of the control myotubes $(n=50)$. Results are presented as mean \pm s.D. ${ }^{*} P<0.05 ;$ SC, scramble siRNA.

Cav1 is abundantly expressed in adipocytes, endothelial cells, type 1 pneumocytes, and smooth muscle cells, and it is the main structural component of caveolae, which are plasma membrane invaginations that function as vesicular transporters for macromolecules and a variety of pathogens by transcytosis or endocytosis (Cohen et al. 2004). In addition, CAV1 functions as a scaffolding protein that recruits numerous signaling molecules to caveolae, thereby activating or inhibiting signaling pathways, depending on the cell-type and signaling molecules (Hansen \& Nichols 2010). Thus, CAV1 is responsible for the pathogenesis of various diseases, including lipodystrophy, atherosclerosis, diabetes, pulmonary fibrosis, and cancer. However, the role of CAV1 in muscle is relatively unstudied. Although the mechanisms of signaldependent regulation in the caveolae remain unclear, our results indicate that IR $\alpha$ and IRS1 are positively regulated by CAV1 in muscle cells. Consistent with these results,

Published by Bioscientifica Ltd 
A

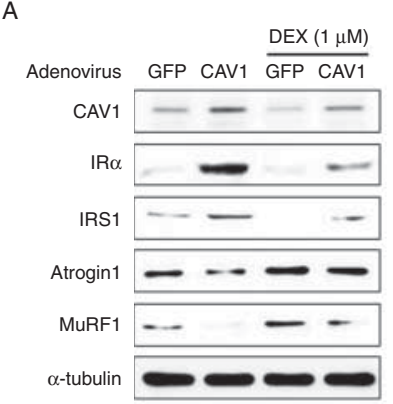

C

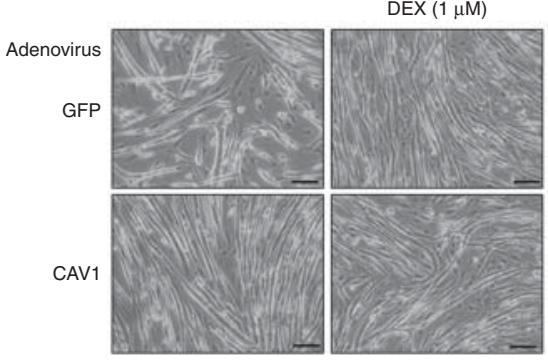

B

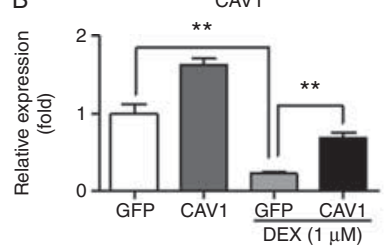

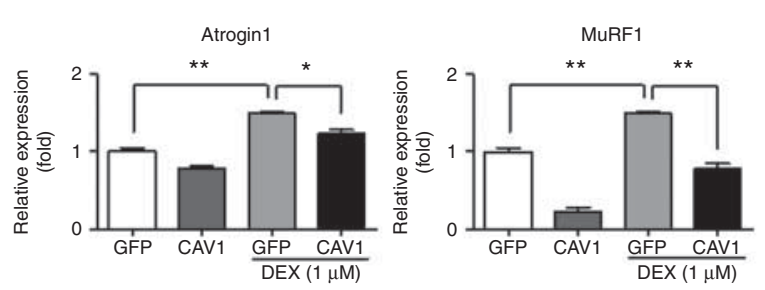

$\mathrm{D}$
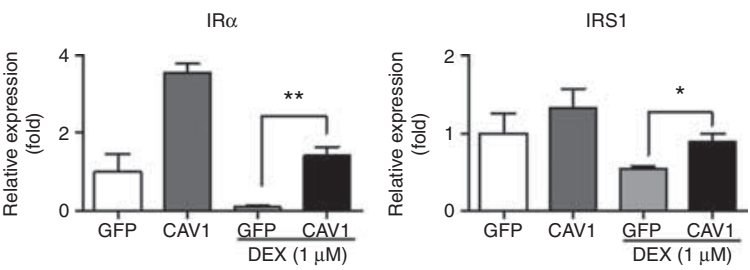

IRS1

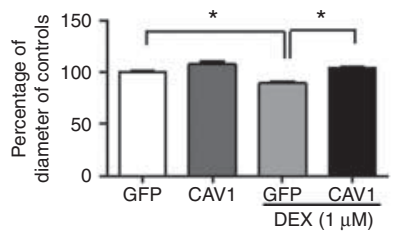

Figure 4

Caveolin-1 overexpression prevents the dexamethasone-induced decrease in myotube diameter. (A) Western blot analysis of $\mathrm{C} 2 \mathrm{C} 12$ myotubes infected with adenovirus expressing Cav1 (AdCav1, $3 \times 10^{11}$ plaque-forming units) or GFP (AdGFP $3 \times 10^{11}$ plaque-forming units) was performed using the indicated antibodies. Myotubes were cultured for $24 \mathrm{~h}$ in the presence or absence of DEX $(1 \mu \mathrm{M})$. (B) Relative band intensities of the western blots from three independent experiments were estimated by densitometric

an increase in Cav1 expression in skeletal muscle improves insulin sensitivity (Cohen et al. 2004, Oh et al. 2008). Conversely, Cav1-null mice displayed skeletal muscle abnormalities due to tubular aggregate formation (Schubert et al. 2007) and exercise intolerance (Razani et al. 2001), demonstrating that insulin/IGF signaling depends on the level of Cav1 expression in muscle.

Cav1-null mice were viable and fertile, but displayed various phenotypes, including abnormal regulation of lipid metabolism and impaired nitric oxide and calcium signaling, which led to aberrant regulation of myogenic tone in the cardiovascular system (Drab et al. 2001) and impaired liver regeneration after injury (Fernandez et al. 2006). On the basis of these observations, homozygotic or heterozygotic mutations in the CAV1 gene have been identified in patients with congenital generalized lipodystrophy type 3 (CGL3) (Kim et al. 2008), partial lipodystrophy, congenital cataracts, neurodegeneration syndrome (LCCNS) (Cao et al. 2008), and primary pulmonary hypertension 3 (PPH3) (Austin et al. 2012). Comparing the clinical features of these syndromes with those of patients who received long-term glucocorticoid quantification. Data are presented as mean \pm s.D. ${ }^{*} * P<0.01$ and $* P<0.05$. (C) Representative photographs of C2C12 myotubes infected with AdCav1 or AdGFP. Scale bars: $100 \mu \mathrm{m}$. (D) Myotubes were stained with PAS and photographed. The diameter of the myotubes from randomly selected fields was measured using ImageJ. Data are expressed as percentages of the diameter of the control cells $(n=50)$. Results are expressed as mean \pm s.D. $* P<0.05$.

therapy indicates that, in addition to muscle wasting and insulin resistance, clinical manifestations such as cataracts, progressive loss of adipose tissue, and hypertension might be caused by glucocorticoid-induced downregulation of Cav1 expression. Moreover, as a nongenomic effect of glucocorticoid, DEX treatment induced rapid phosphorylation of CAV1, which is required for activation of the PI3K-AKT pathway in the glucocorticoid-mediated growth arrest of A549 cells (Matthews et al. 2008). Therefore, these observations clearly support our findings that CAV1 is a key downstream effector of glucocorticoids.

CAV3, a muscle-specific isoform, plays a role in muscle development and physiology by forming a complex with dystrophin and glycoprotein, thereby stabilizing the sarcolemma. Thus, CAV3 deficiency due to mutations in the gene causes the degeneration of myofibrils, leading to skeletal muscle diseases (Gazzerro et al. 2010). Results of functional analyses of CAV3-null mice indicated that CAV3 is an enhancer of insulin signaling (Oshikawa et al. 2004). In addition, CAV3 inhibits myostatin signaling through its interaction with ALK5, resulting in increased myofiber size (Ohsawa et al. 2004). These findings indicate

Published by Bioscientifica Ltd 


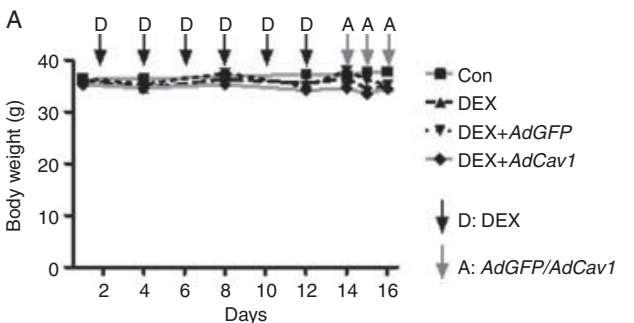

C
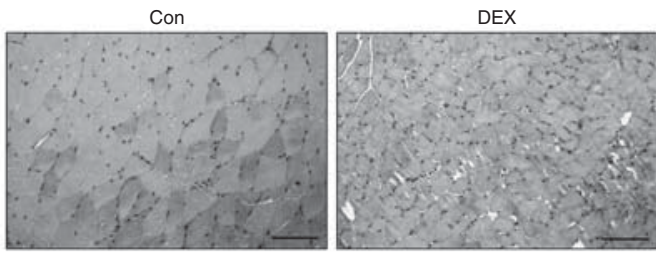

D

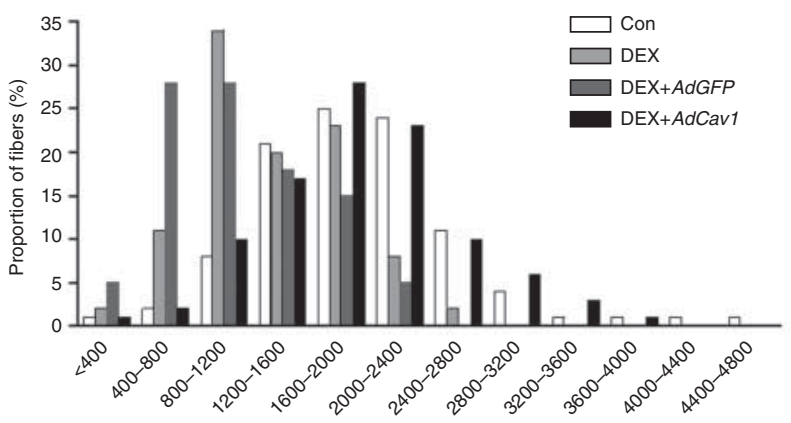

Cross sectional area $\left(\mu \mathrm{m}^{2}\right)$
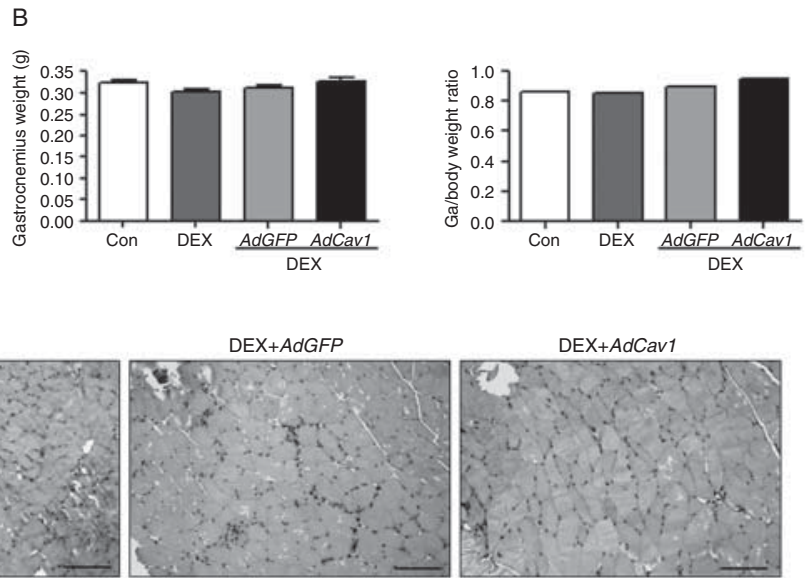

$E$

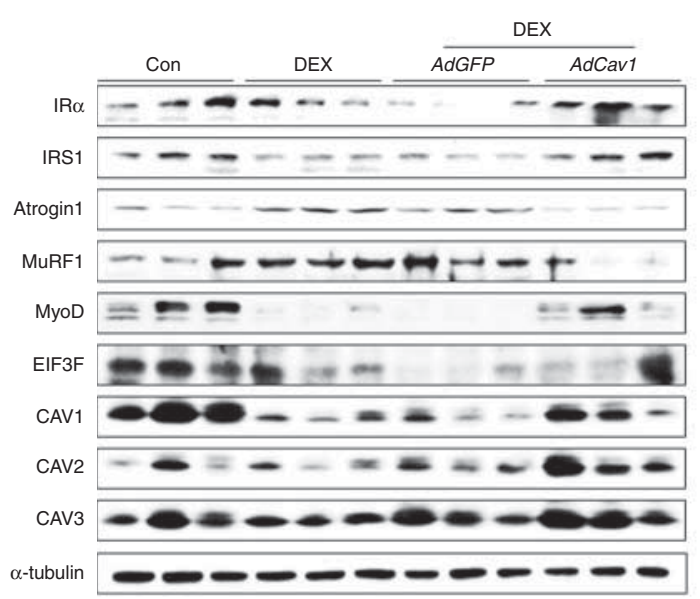

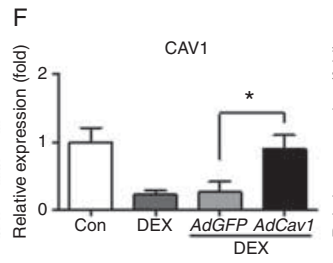
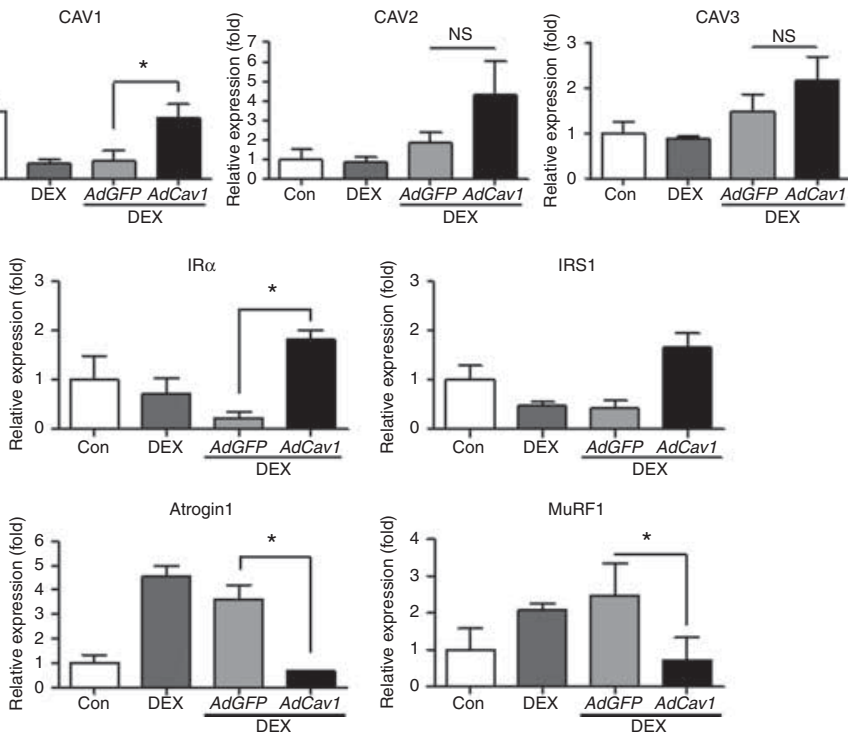

\section{Figure 5}

Caveolin-1 overexpression restores dexamethasone-induced muscle atrophy in mice. C57BL/6 mice were treated every other day with injections of saline (CON) or dexamethasone (DEX; $5 \mathrm{mg} / \mathrm{kg}$ ) for 12 days. Subsequently, received daily injections of AdCav1 (DEX-AdCav1) or AdGFP virus (DEX$A d G F P$ ) into the gastrocnemius muscle at eight sites for 3 days. Mice were killed on the 16th day. (A) Total body weight. (B) Gastrocnemius mass and ratio of weight of gastrocnemius (Ga) to total body weight, $n=5$ for each group. (C) Representative photographs of AdCav1 or AdGFP-injected gastrocnemius muscles from DEX-treated mice. The sections were stained with hematoxylin and eosin (H\&E). Scale bars: $200 \mu \mathrm{m}$. (D) The distribution of muscle fibers with different cross-sectional area (CSA). Two hundred fibers per gastrocnemius muscle were randomly chosen and the CSA was measured using a microscope. (E) Western blot analysis of gastrocnemius muscle using the indicated antibodies. (F) Relative band intensities from five mice were estimated by densitometric quantification. Data are presented as mean \pm s.D. ${ }^{*} P<0.01$.

Published by Bioscientifica Ltd 
that CAV3 could be a plausible target for glucocorticoids. However, contrary to expectations, our results indicated that CAV3 protein in mouse skeletal muscle was increased, although the increase was not statistically significant rather than decreased in response to DEX treatment. Interestingly, overexpression of Cav3 also induces muscle fiber degeneration by downregulating dystrophin (Galbiati et al. 1999). Taken together, these results indicate that both increased Cav3 expression and decreased Cav1 expression are likely to contribute to DEX-mediated muscle atrophy.

The transcriptional regulation of specific genes by glucocorticoids is dependent on specific GREs. It is well established that GREs have both positive and negative regulatory functions (Dostert \& Heinzel 2004). Positive GREs activate transcription of related genes, while negative GREs (nGREs) inhibit transcription. Results described in reports indicated that DEX induced Cav1 expression at both the mRNA and protein levels in endothelial cells from thoracic aorta and lung artery (Igarashi et al. 2013). Although CAV1 induction by DEX was glucocorticoidreceptor-dependent, specific GREs responsible for CAV1 induction in the endothelial cells were not identified. In contrast, our results indicated that DEX suppresses Cav1 expression, and that a nGRE in the Cav1 gene located $387 \mathrm{bp}$ upstream of the promoter region is responsible for DEX-induced suppression of Cav1 gene expression by sitedirected mutagenesis. Results of gene expression analyses using microarrays indicated that a number of genes are downregulated by glucocorticoids (van Batenburg et al. 2010). However, nGREs have been identified only in the promoter region of a limited number of genes, because nGREs do not have a highly conserved sequence. Moreover, nGREs exhibit an inhibitory effect on the transcription of target genes in a cell-type-specific manner (Turney \& Kovacs 2001), indicating that unidentified transcription factors such as coactivators or corepressors may be involved in the regulation of DEX-glucocorticoid receptor-GRE interaction. This idea is supported by the fact that DEX and corticosterone induced different muscle wasting responses in cultured myotubes. Moreover, the effects of RU38486, an antagonist of glucocorticoid receptor, depended on the muscle cell line used (Menconi et al. 2008). Thus, further studies are required to elucidate the physiological and molecular mechanisms of tissue-specific suppression by nGREs.

In summary, our results indicated that Cav1 is negatively regulated by DEX through GR targeting of nGREs in the promoter region of the gene, thereby mediating DEXinduced muscle atrophy through the inhibition of insulin signaling. Thus, these results define a new pathway linking glucocorticoid signaling and the insulin pathway in the induction of muscle atrophy, and provide a useful target that could be exploited to prevent muscle atrophy.

\section{Supplementary data}

This is linked to the online version of the paper at http://dx.doi.org/10.1530/ JOE-14-0490.

\section{Declaration of interest}

The authors declare that there is no conflict of interest that could be perceived as prejudicing the impartiality of the research reported.

\section{Funding}

This work was supported by grants from the National Research Foundation of Korea (NRF-2010-0019472), Bio \& Medical Technology Development Program (NRF-2011-0030133), and the Korea Healthcare Technology R\&D project, Ministry of Health and Welfare (grant number A100190). Y H S, S-J L, K-B L, and $\mathrm{J}-\mathrm{H} \mathrm{L}$ were supported by the graduate program of the brain Korea 21 (BK21) plus program, Korean Ministry of Education, Science and Technology.

\section{References}

Attaix D \& Baracos VE 2010 MAFbx/Atrogin-1 expression is a poor index of muscle proteolysis. Current Opinion in Clinical Nutrition and Metabolic Care 13 223-224. (doi:10.1097/MCO.0b013e328338b9a6)

Austin ED, Ma L, LeDuc C, Berman Rosenzweig E, Borczuk A, Phillips JA III, Palomero T, Sumazin P, Kim HR, Talati MH et al. 2012 Whole exome sequencing to identify a novel gene (caveolin-1) associated with human pulmonary arterial hypertension. Circulation. Cardiovascular Genetics 5 336-343. (doi:10.1161/CIRCGENETICS.111.961888)

Baehr LM, Furlow JD \& Bodine SC 2011 Muscle sparing in muscle RING finger 1 null mice: response to synthetic glucocorticoids. Journal of Physiology 589 4759-4776. (doi:10.1113/jphysiol.2011.212845)

van Batenburg MF, Li H, Polman JA, Lachize S, Datson NA, Bussemaker HJ \& Meijer OC 2010 Paired hormone response elements predict caveolin-1 as a glucocorticoid target gene. PLoS ONE 5 e8839. (doi:10.1371/journal.pone.0008839)

Bonaldo P \& Sandri M 2013 Cellular and molecular mechanisms of muscle atrophy. Disease Models \& Mechanisms 6 25-39. (doi:10.1242/dmm. 010389)

Boscher C \& Nabi IR 2012 Caveolin-1: role in cell signaling. Advances in Experimental Medicine and Biology 729 29-50. (doi:10.1007/978-1-46141222-9_3)

Cao H, Alston L, Ruschman J \& Hegele RA 2008 Heterozygous Cav1 frameshift mutations (MIM 601047) in patients with atypical partial lipodystrophy and hypertriglyceridemia. Lipids in Health and Disease 73. (doi:10.1186/1476-511X-7-3)

Clarke BA, Drujan D, Willis MS, Murphy LO, Corpina RA, Burova E, Rakhilin SV, Stitt TN, Patterson C, Latres E et al. 2007 The E3 ligase MuRF1 degrades myosin heavy chain protein in dexamethasonetreated skeletal muscle. Cell Metabolism 6 376-385. (doi:10.1016/ j.cmet.2007.09.009)

Cohen AW, Hnasko R, Schubert W \& Lisanti MP 2004 Role of caveolae and caveolins in health and disease. Physiological Reviews 84 1341-1379. (doi:10.1152/physrev.00046.2003)

Published by Bioscientifica Ltd 
Dostert A \& Heinzel T 2004 Negative glucocorticoid receptor response elements and their role in glucocorticoid action. Current Pharmaceutical Design 10 2807-2816. (doi:10.2174/1381612043383601)

Drab M, Verkade P, Elger M, Kasper M, Lohn M, Lauterbach B, Menne J, Lindschau C, Mende F, Luft FC et al. 2001 Loss of caveolae, vascular dysfunction, and pulmonary defects in caveolin-1 gene-disrupted mice. Science 293 2449-2452. (doi:10.1126/science.1062688)

Fernandez MA, Albor C, Ingelmo-Torres M, Nixon SJ, Ferguson C, Kurzchalia T, Tebar F, Enrich C, Parton RG \& Pol A 2006 Caveolin-1 is essential for liver regeneration. Science 313 1628-1632. (doi:10.1126/ science.1130773)

Galbiati F, Volonte D, Minetti C, Chu JB \& Lisanti MP 1999 Phenotypic behavior of caveolin-3 mutations that cause autosomal dominant limb girdle muscular dystrophy (LGMD-1C). Retention of LGMD-1C caveolin-3 mutants within the golgi complex. Journal of Biological Chemistry 274 25632-25641. (doi:10.1074/jbc.274.36.25632)

Gazzerro E, Sotgia F, Bruno C, Lisanti MP \& Minetti C 2010 Caveolinopathies: from the biology of caveolin-3 to human diseases. European Journal of Human Genetics 18 137-145. (doi:10.1038/ejhg.2009.103)

Glass DJ 2010 Signaling pathways perturbing muscle mass. Current Opinion in Clinical Nutrition and Metabolic Care 13 225-229. (doi:10.1097/MCO. Ob013e32833862df)

Hansen CG \& Nichols BJ 2010 Exploring the caves: cavins, caveolins and caveolae. Trends in Cell Biology 20 177-186. (doi:10.1016/j.tcb.2010. 01.005)

Igarashi J, Hashimoto T, Shoji K, Yoneda K, Tsukamoto I, Moriue T, Kubota Y \& Kosaka H 2013 Dexamethasone induces caveolin-1 in vascular endothelial cells: implications for attenuated responses to VEGF. American Journal of Physiology. Cell Physiology 304 C790-C800. (doi:10.1152/ajpcell.00268.2012)

Kim CA, Delepine M, Boutet E, El Mourabit H, Le Lay S, Meier M, Nemani M, Bridel E, Leite CC, Bertola DR et al. 2008 Association of a homozygous nonsense caveolin-1 mutation with Berardinelli-Seip congenital lipodystrophy. Journal of Clinical Endocrinology and Metabolism 93 1129-1134. (doi:10.1210/jc.2007-1328)

Kim HS, Kim HJ, Kim YS, Park SC, Harris R \& Kim CK 2009 Caveolin, GLUT4 and insulin receptor protein content in human arm and leg muscles. European Journal of Applied Physiology 106 173-179. (doi:10.1007/ s00421-009-1001-1)

Koh A, Lee MN, Yang YR, Jeong H, Ghim J, Noh J, Kim J, Ryu D, Park S, Song P et al. $2013 \mathrm{C} 1-$ Ten is a protein tyrosine phosphatase of insulin receptor substrate 1 (IRS-1), regulating IRS-1 stability and muscle atrophy. Molecular and Cellular Biology 33 1608-1620. (doi:10.1128/ MCB.01447-12)

Kuo T, Harris CA \& Wang JC 2013 Metabolic functions of glucocorticoid receptor in skeletal muscle. Molecular and Cellular Endocrinology $\mathbf{3 8 0}$ 79-88. (doi:10.1016/j.mce.2013.03.003)

Matthews L, Berry A, Ohanian V, Ohanian J, Garside H \& Ray D 2008 Caveolin mediates rapid glucocorticoid effects and couples glucocorticoid action to the antiproliferative program. Molecular Endocrinology 22 1320-1330. (doi:10.1210/me.2007-0154)

Menconi M, Fareed M, O’Neal P, Poylin V, Wei W \& Hasselgren PO 2007 Role of glucocorticoids in the molecular regulation of muscle wasting. Critical Care Medicine 35 S602-S608. (doi:10.1097/01.CCM. 0000279194.11328.77)

Menconi M, Gonnella P, Petkova V, Lecker S \& Hasselgren PO 2008 Dexamethasone and corticosterone induce similar, but not identical, muscle wasting responses in cultured L6 and C2C12 myotubes. Journal of Cellular Biochemistry 105 353-364. (doi:10.1002/jcb.21833)
Oh YS, Khil LY, Cho KA, Ryu SJ, Ha MK, Cheon GJ, Lee TS, Yoon JW, Jun HS \& Park SC 2008 A potential role for skeletal muscle caveolin-1 as an insulin sensitivity modulator in ageing-dependent non-obese type 2 diabetes: studies in a new mouse model. Diabetologia 51 1025-1034. (doi:10.1007/s00125-008-0993-0)

Oh YS, Lee TS, Cheon GJ, Jang IS, Jun HS \& Park SC 2011 Modulation of insulin sensitivity and caveolin-1 expression by orchidectomy in a nonobese type 2 diabetes animal model. Molecular Medicine 17 4-11. (doi:10.2119/molmed.2009.00105)

Ohsawa Y, Toko H, Katsura M, Morimoto K, Yamada H, Ichikawa Y, Murakami T, Ohkuma S, Komuro I \& Sunada Y 2004 Overexpression of P104L mutant caveolin-3 in mice develops hypertrophic cardiomyopathy with enhanced contractility in association with increased endothelial nitric oxide synthase activity. Human Molecular Genetics 13 151-157. (doi:10.1093/hmg/ddh014)

Oshikawa J, Otsu K, Toya Y, Tsunematsu T, Hankins R, Kawabe J, Minamisawa S, Umemura S, Hagiwara Y \& Ishikawa Y 2004 Insulin resistance in skeletal muscles of caveolin-3-null mice. PNAS 101 12670-12675. (doi:10.1073/pnas.0402053101)

Polge C, Heng AE, Jarzaguet M, Ventadour S, Claustre A, Combaret L, Bechet D, Matondo M, Uttenweiler-Joseph S, Monsarrat B et al. 2011 Muscle actin is polyubiquitinated in vitro and in vivo and targeted for breakdown by the E3 ligase MuRF1. FASEB Journal 25 3790-3802. (doi:10.1096/fj.11-180968)

Razani B, Engelman JA, Wang XB, Schubert W, Zhang XL, Marks CB, Macaluso F, Russell RG, Li M, Pestell RG et al. 2001 Caveolin-1 null mice are viable but show evidence of hyperproliferative and vascular abnormalities. Journal of Biological Chemistry 276 38121-38138.

Razani B, Combs TP, Wang XB, Frank PG, Park DS, Russell RG, Li M, Tang B, Jelicks LA, Scherer PE et al. 2002 Caveolin-1-deficient mice are lean, resistant to diet-induced obesity, and show hypertriglyceridemia with adipocyte abnormalities. Journal of Biological Chemistry 277 8635-8647. (doi:10.1074/jbc.M110970200)

Schakman O, Gilson H \& Thissen JP 2008 Mechanisms of glucocorticoidinduced myopathy. Journal of Endocrinology 197 1-10. (doi:10.1677/ JOE-07-0606)

Schakman O, Kalista S, Barbe C, Loumaye A \& Thissen JP 2013 Glucocorticoid-induced skeletal muscle atrophy. International Journal of Biochemistry \& Cell Biology 45 2163-2172. (doi:10.1016/j.biocel.2013. 05.036)

Schubert W, Sotgia F, Cohen AW, Capozza F, Bonuccelli G, Bruno C, Minetti C, Bonilla E, Dimauro S \& Lisanti MP 2007 Caveolin $1^{(-/-)}$- and caveolin $2^{(-l-)}$-deficient mice both display numerous skeletal muscle abnormalities, with tubular aggregate formation. American Journal of Pathology 170 316-333. (doi:10.2353/ajpath.2007.060687)

Shimizu N, Yoshikawa N, Ito N, Maruyama T, Suzuki Y, Takeda S, Nakae J, Tagata Y, Nishitani S, Takehana K et al. 2011 Crosstalk between glucocorticoid receptor and nutritional sensor mTOR in skeletal muscle. Cell Metabolism 13 170-182. (doi:10.1016/j.cmet.2011.01.001)

Turney MK \& Kovacs WJ 2001 Function of a truncated glucocorticoid receptor form at a negative glucocorticoid response element in the proopiomelanocortin gene. Journal of Molecular Endocrinology 26 43-49. (doi:10.1677/jme.0.0260043)

Zhao W, Qin W, Pan J, Wu Y, Bauman WA \& Cardozo C 2009 Dependence of dexamethasone-induced Akt/FOXO1 signaling, upregulation of $\mathrm{MAFbx}$, and protein catabolism upon the glucocorticoid receptor. Biochemical and Biophysical Research Communications 378 668-672. (doi:10.1016/j.bbrc.2008.11.123)

Received in final form 2 February 2015

Accepted 12 February 2015

Accepted Preprint published online 16 February 2015 http://joe.endocrinology-journals.org

DOI: $10.1530 / J O E-14-0490$
(C) 2015 The authors Printed in Great Britain
Published by Bioscientifica Ltd 